\title{
11. PETROLOGY AND CHEMISTRY OF BASALTS FROM THE NAZCA PLATE: PART 1-PETROGRAPHY AND MINERAL CHEMISTRY
}

\author{
Colin H. Donaldson, Lunar Science Institute, Houston, Texas, and University of St. Andrews, Scotland \\ Roy W. Brown, Lockheed Electronics Corporation, Houston, Texas \\ and \\ Arch M. Reid, NASA Johnson Space Center, Houston, Texas
}

\section{INTRODUCTION}

We report on the petrography and mineral chemistry of 21 basalt samples drilled during Leg 34 of the Deep Sea Drilling Project from the Nazca plate. The suite of samples comprises 13 from Hole 319A, 3 from Hole $320 \mathrm{~B}$, and 5 from Site 321. The same samples have also been analyzed for major, minor, and trace element contents (Rhodes et al., this volume). Results of petrographic observations and electron microprobe analyses are summarized in Tables 1-6 and Figures 1-10.

\section{RESULTS}

Table 1 lists the catalog numbers of the samples and their locations in cooling units and summarizes the rock types, textures, and range of compositions for the three principal phases-plagioclase, augite, and olivine. Rock types are distinguished on the basis of the groundmass grain size (medium grained [1-5 mm] versus fine grained $[<1 \mathrm{~mm}]$ ) and phenocryst assemblage. Many of the rocks contain phenocrysts, the most abundant of which is plagioclase, followed by olivine. One sample, 319A-6$1,84-89 \mathrm{~cm}$, contains pyroxene microphenocrysts. In the groundmass, elongate (in some cases, skeletal) plagioclase laths are set in a cryptocrystalline matrix, or form an ophitic or intergranular texture with pyroxene, or form an intersertal texture. All three of these textures coexist in some samples (e.g., 319A-4-1, 129-132 cm). Groundmass olivine is present only in the finer grained rocks from Holes 319A and 320B and is totally absent in samples from Site 321. The groundmass olivines are subophitically enclosed by plagioclase laths (Figure 4). They are particularly susceptible to alteration to dark brown smectite. Each olivine grain may be either completely replaced or mantled by smectite (Figure 4). Those rocks in Table 1 for which the groundmass olivine column is labelled "none" contain neither groundmass crystals nor smectite crystals which could be inferred to be pseudomorphs after olivine. None of the samples contained glass. However, samples with intersertal texture contain abundant smectite, assumed to be the alteration product of glass and its associated devitrification products. Therefore, despite the low water contents and oxidation ratios of the Leg 34 basalts (Hart et al., 1974; R hodes et al., this volume), relative to most other basalts recovered by the Deep Sea Drilling Project, the rocks are not mineralogically fresh.

Plagioclase phenocrysts have two types of occurrences-either isolated in the groundmass or, more commonly in glomerophyric units (Figure 5), some of which also contain olivine. The cores of these crystals $\left(\mathrm{An}_{87-68}\right)$ are either unzoned or are oscillatory zoned with adjacent zones differing by 1-4 mol percent An. The margins of crystals in contact with the groundmass are continuously normal zoned. The periphery of each phenocryst has the same composition as the margins of groundmass plagioclase laths, which are always normally zoned from core $\left(\mathrm{An}_{75^{-63}}\right)$ to $\mathrm{rim}\left(\mathrm{An}_{61-27}\right)$. Fe content of both phenocryst and groundmass plagioclase is substantial (i.e., 0.3-1.0 wt\%) and increases with $\mathrm{Ab}$ content of the plagioclase (Table 3). Plagioclase zoning is much more extensive in medium-grained rocks than in finegrained rocks (Table 1).

Olivine phenocrysts have limited ranges of core composition (Fo89-84, Tables 1 and 2). An unzoned core is typically surrounded by a narrow normally zoned mantle. Groundmass olivines are slightly zoned (Table 2) and generally are 1-3 mol \% less forsterite-rich than the core of the phenocrysts in the same rock. The $\mathrm{CaO}$ contents of both phenocrystic and groundmass olivines exceed $1000 \mathrm{ppm}$ (Table 2). The Ca content tends to increase with fayalite content of the olivine.

All the pyroxenes studied are augites. At Hole 319A and Site 321, pyroxenes have wide ranges of composition (Figure 10 and Table 4). Pyroxene in the rocks from Hole $320 \mathrm{~B}$ is very fine grained and only three grains, all in one sample, were analyzed (Table 4). Augite compositions from individual rocks plotted in the pyroxene quadrilateral define either quench trends (cf Evans and Moore, 1968) or trends subparallel to, and slightly less calcic than, the Skaergaard trend. Some single ophitic crystals in the coarse-grained rocks are extensively zoned with respect to $\mathrm{Fe}-\mathrm{Mg}$ content (Table 4). The microphenocrysts in Sample 319A-6-1, 84-89 cm are similar in major element composition to the earliest augite to crystallize in the Skaergaard intrusion (Figure 10).

The opaque phases are Fe-Ti oxides that occur in the groundmass, commonly as very small skeletal or dendritic crystallites. Titanomagnetite with around $25 \mathrm{wt} \%$ $\mathrm{TiO}_{2}$ is present in virtually all of the basalts and is accompanied by ilmenite in more than half of the samples (Table 5). Only one sample (320B-3-1, 120-125 cm) has a small euhedral $\mathrm{Mg}$-Al-Cr spinel grain (Table 6) which is enclosed by early plagioclase. All other samples lack a Cr-spinel phase, a deficiency which is atypical of most mid-ocean-ridge basalts.

\section{CONCLUSIONS}

Basalts at Site 321 lack both phenocryst and groundmass olivine (Table 1; contrast Holes 319A and 320B); also, the plagioclase phenocrysts are much less calcic 
TABLE 1

Summary of Samples Investigated, Rock Types Present and Their Textures, and the Range of Phase Composition in Each Rock

\begin{tabular}{|c|c|c|c|c|c|c|c|c|c|c|c|c|c|c|}
\hline \multirow[b]{2}{*}{$\begin{array}{c}\text { Sample } \\
\text { (Interval in cm) }\end{array}$} & \multirow[b]{2}{*}{$\begin{array}{l}\text { Cooling } \\
\text { Unit }^{\mathrm{a}}\end{array}$} & \multirow[b]{2}{*}{ Rock Type } & \multirow{2}{*}{$\begin{array}{l}\text { Olivine } \\
\text { Phenocryst } \\
\text { (Fo mol \%) }\end{array}$} & \multirow{2}{*}{$\begin{array}{l}\text { Olivine } \\
\text { Groundmass } \\
\text { (Fo mol \%) }\end{array}$} & \multicolumn{3}{|c|}{ Plagioclase Phenocryst (Core) ${ }^{\mathrm{c}}$} & \multicolumn{3}{|c|}{ Plagioclase Groundmass ${ }^{\mathrm{d}}$} & \multicolumn{3}{|c|}{ Augite $^{\mathrm{e}}$} & \multirow[b]{2}{*}{ Comments } \\
\hline & & & & & An & $\underset{(\mathrm{mol} \%)}{\mathrm{Ab}}$ & Or & An & $\underset{(\mathrm{mol} \%)}{\mathrm{Ab}}$ & Or & $\mathrm{Ca}$ & $\begin{array}{c}\mathrm{Mg} \\
\text { (atomic props.) }\end{array}$ & $\mathrm{Fe}$ & \\
\hline $319 \mathrm{~A}-1-1,138-142$ & 2 & Fine-grained basalt & None & 85 & & & & $73.1-65.4$ & $26.8-34.3$ & $0.1-0.3$ & $33-35.8$ & $58-49.8$ & $8.7-14.4$ & $\begin{array}{l}\text { Skeletal olivine }(0.1 \mathrm{~mm} \text { across) and } \\
\text { glomerophyric units of plagioclase } \\
(0.5 \times 0.05 \mathrm{~mm}) \text { subophitically } \\
\text { enclosed by augite }(0.1 \mathrm{~mm} \text { across }) \\
\text { set in cryptocrystalline matrix (fig. } 1)\end{array}$ \\
\hline 319A-2-2, 114-117 & 3 & $\begin{array}{l}\text { Medium-grained } \\
\text { feldspar phyric } \\
\text { basalt }\end{array}$ & None & None & $81.9-73.4$ & $17.9-26.3$ & $0.2-0.3$ & $63.8-34.8$ & $35.9-64.6$ & $0.3-0.6$ & $34.6-43.6$ & $40.4-43.4$ & $25.0-13.0$ & $\begin{array}{l}\text { Plagioclase phenocrysts and glomero- } \\
\text { crysts set in groundmass of intergran- } \\
\text { ular and intersertal texture }\end{array}$ \\
\hline $319 \mathrm{~A}-3-2,14-17$ & 3 & $\begin{array}{l}\text { Medium-grained } \\
\text { feldspar phyric } \\
\text { basalt }\end{array}$ & None & None & $74.8-69.6$ & 24.9-29.9 & $0.3-0.5$ & 64.4-32.6 & $35.3-66.7$ & $0.3-0.7$ & $35.4-41.9$ & $52-46.7$ & 12.6-11.3 & $\begin{array}{l}\text { Plagioclase phenocrysts (up to } 1.5 \\
\text { mm across) and glomerocrysts set in } \\
\text { groundmass of poikilophitic and } \\
\text { intersertal tex tures (fig. 2) }\end{array}$ \\
\hline $319 \mathrm{~A}-3-2,127-130$ & 3 & $\begin{array}{l}\text { Medium-grained } \\
\text { feldspar phyric } \\
\text { basalt }\end{array}$ & None & None & $78.8-67.5$ & $21.0-32.1$ & $0.2-0.4$ & $66.0-36.0$ & $33.6-63.3$ & $0.4-0.7$ & $40-36$ & $50-37$ & $10-27$ & $\begin{array}{l}\text { Tex ture similar to Sample } 319 \mathrm{~A}-3-2 \text {, } \\
14-17 \mathrm{~cm}\end{array}$ \\
\hline $319 \mathrm{~A}-3-3,46-49$ & 3 & $\begin{array}{l}\text { Medium-grained } \\
\text { feldspar phyric } \\
\text { basalt }\end{array}$ & None & None & $85.1-76.9$ & $14.6-22.9$ & $0.3-0.2$ & 73.2-31.7 & $26.4-67.5$ & $0.4-0.7$ & $41.3-36.5$ & $43.6-50.8$ & $15.1-12.8$ & $\begin{array}{l}\text { Tex ture similar to Sample 319A-3-2, } \\
14-17 \mathrm{~cm}\end{array}$ \\
\hline $319 \mathrm{~A}-3-3,106-109$ & 3 & $\begin{array}{l}\text { Medium-grained } \\
\text { feldspar phyric } \\
\text { basalt }\end{array}$ & None & None & $77.8-72.5$ & $22.0-27.4$ & 0.2 & $65.7-37.0$ & $34.0-62.6$ & $0.3-0.4$ & $41-31.3$ & $46-44.1$ & $13-24.6$ & $\begin{array}{l}\text { Texture similar to Sample 319A-3-2, } \\
14-17 \mathrm{~cm}\end{array}$ \\
\hline $319 \mathrm{~A}-3-4,18-21$ & 4 & $\begin{array}{l}\text { Medium-grained } \\
\text { olivine feldspar } \\
\text { phyric basalt }\end{array}$ & $85.4-71$ & $80.0-76.0$ & $85.0-80.1$ & $14.7-19.8$ & $0.3-0.1$ & $66.3-27.3$ & $33.5-72.0$ & $0.2-0.7$ & $39-32$ & $44.7-43.5$ & 16.1-24.5 & $\begin{array}{l}\text { Olivine phenocrysts (up to } 3 \mathrm{~mm} \\
\text { across), plagioclase phenocrysts (up } \\
\text { to } 1.5 \mathrm{~mm} \text { across) plagioclase glomero- } \\
\text { crysts, and olivine-plagioclase glo- } \\
\text { merocrysts (troctolite, fig. } 3 \text { ) set in } \\
\text { Groundmass textures identical to } \\
\text { Sample } 319 \mathrm{~A}-3-2,14-17 \mathrm{~cm}\end{array}$ \\
\hline $319 A-3-4,100-103$ & 4 & $\begin{array}{l}\text { Medium-grained } \\
\text { feldspar phyric } \\
\text { basalt }\end{array}$ & None & None & 77.9 & 21.9 & 0.2 & 75.4-33.2 & $24.3-66.2$ & $0.3-0.6$ & $43.2-34.2$ & $43.9-48.7$ & 12.9-17.1 & \\
\hline $319 A-3-5,37-41$ & 5 & $\begin{array}{l}\text { Medium-grained } \\
\text { olivine feldspar } \\
\text { phyric basalt }\end{array}$ & 88.8-79.5 & None & $80.5-75.1$ & 9.3-24.7 & 0.2 & $65.4-30.6$ & $34.2-68.8$ & $0.3-0.6$ & $41-37.3$ & $47.2-45.2$ & 11.7-17.6 & $\begin{array}{l}\text { Tex ture similar to Sample 319A-3-2, } \\
14-17 \mathrm{~cm}\end{array}$ \\
\hline $319 \mathrm{~A}-4-1,129-132$ & 5 & $\begin{array}{l}\text { Fine-grained } \\
\text { olivine feldspar } \\
\text { basalt }\end{array}$ & 88-79 & 81.3 & $80.5-77.9$ & $9.4-22.0$ & $0.1-0.2$ & $65.8-34.2$ & $33.8-65.1$ & $0.4-0.8$ & $44.2-29.4$ & $44.2-43$ & $11.1-27.5$ & $\begin{array}{l}\text { Olivine phenocrysts (up to } 3 \mathrm{~mm} \\
\text { across), plagioclase phenocrysts (up } \\
\text { to } 1.3 \mathrm{~mm} \text { across), plagioclase glo- } \\
\text { merocrysts, and olivine-plagioclase } \\
\text { glomerocrysts (troctolite fragments) } \\
\text { set in groundmass of ophitic, inter- } \\
\text { granular and intersertal textures; } \\
\text { Small grains of olivine scattered } \\
\text { through matrix (fig. 4) }\end{array}$ \\
\hline $319 \mathrm{~A}-5-1,76-79$ & 5 & $\begin{array}{l}\text { Fine-grained } \\
\text { olivine feldspar }\end{array}$ & $83.5-80.5$ & 81.1 & $86.7-74.6$ & $3.0-25.2$ & $0.2-0.1$ & $63.3-39.2$ & $36.4-60.3$ & $0.3-0.5$ & $41.2-36.2$ & $47.7-41.6$ & $11.1-22.2$ & $\begin{array}{l}\text { Tex ture similar to Sample 319A-4-1, } \\
129-132 \mathrm{~cm}\end{array}$ \\
\hline $319 \mathrm{~A}-6-1,84-89$ & 7 & $\begin{array}{l}\text { Fine-grained } \\
\text { pyroxene feldspar } \\
\text { phyric basalt }\end{array}$ & None & None & $82.1-70.6$ & $17.7-29.1$ & $0.2-0.3$ & $63.3-60.0$ & $36.3-39.6$ & 0.4 & $\begin{array}{l}41.5 \\
\text { (phenocrys } \\
\text { mass pyrox }\end{array}$ & $\begin{array}{l}49.7 \\
\text { sts only analyzed; } \\
\text { kene too small for }\end{array}$ & $\begin{array}{l}8.7 \\
\text {; ; iround- } \\
\text { ir analy sis) }\end{array}$ & $\begin{array}{l}\text { Plagioclase phenocrysts (up to } 3 \times 1 \\
\text { mm) and glomerocrysts (fig. 5) and } \\
\text { pyroxene glomerocrysts (up to } 0.3 \\
\text { mm across, fig. 6) set in groundmass } \\
\text { of felted plagioclase laths, crypto- } \\
\text { crystalline material, and altered glass }\end{array}$ \\
\hline $319 \mathrm{~A}-7-1,119-122$ & 8 & $\begin{array}{l}\text { Fine-grained } \\
\text { olvine feldspar } \\
\text { phyric basalt }\end{array}$ & $84.0-81.5$ & 83.0 & $78.8-73.2$ & $21.0-26.6$ & 0.2 & 69.1-39.6 & $30.7-59.9$ & $0.2-0.5$ & 42 & $48-43$ & $10-15$ & $\begin{array}{l}\text { Tex ture similar to Sample 319A-5-1, } \\
76-79 \mathrm{~cm}\end{array}$ \\
\hline
\end{tabular}




\begin{tabular}{|c|c|c|c|c|c|c|c|c|c|c|c|c|c|}
\hline 320B-3-1, 120-125 & $\begin{array}{l}\text { Fine-grained } \\
\text { olivine feldspar } \\
\text { microphyric basalt }\end{array}$ & None & $85.0-81.0$ & 76.3 & 23.4 & 0.3 & $71.2-61.3$ & $28.5-38.0$ & $0.4-0.7$ & \multicolumn{3}{|c|}{ No pyroxene } & $\begin{array}{l}\text { Plagioclase microphenocrysts (up to } 1 \\
\mathrm{~mm} \text { across), skeletal olivines }(0.5 \mathrm{~mm}) \\
\text { and glomerophyric units of plagio- } \\
\text { clase laths }(1 \times 0.1 \mathrm{~mm}) \text { subophiti- } \\
\text { cally enclosed by olivine }(0.5 \mathrm{~mm} \\
\text { across) set in cryptocrystalline } \\
\text { groundmass }\end{array}$ \\
\hline $320 \mathrm{~B}-4-1,130-133$ & $\begin{array}{l}\text { Fine-grained } \\
\text { feldspar microphyric } \\
\text { basalt }\end{array}$ & None & None & $69.7-67.2$ & 29.9-32.5 & 0.4 & $68.4-46.9$ & $31.2-52.4$ & $0.4-0.8$ & $37.5-30$ & $46.5-54$ & 16 & $\begin{array}{l}\text { Skeletal plagioclase microphenocrysts } \\
\text { (up to } 1 \times 0.3 \mathrm{~mm}) \text { set in groundmass } \\
\text { of felted plagioclase laths }(0.2 \times 0.05 \\
\mathrm{mm} \text { ) and cryptocrystalline material } \\
\text { (fig. 7) }\end{array}$ \\
\hline 320B-5-1, 117-123 & $\begin{array}{l}\text { Fine-grained } \\
\text { feldspar phyric } \\
\text { basalt }\end{array}$ & None & None & 69.4 & 30.1 & 0.2 & $70.7-49.1$ & $29.1-50.0$ & $0.3-0.9$ & & \multicolumn{2}{|l|}{ No pyroxene } & $\begin{array}{l}\text { Texture similar to Sample 320B-4-1, } \\
130-133 \mathrm{~cm} \text {, but plagioclase pheno- } \\
\text { crysts are euhedral, some form } \\
\text { glomerocrysts }\end{array}$ \\
\hline $321-13-4,119-124$ & $\begin{array}{l}\text { Fine-grained } \\
\text { feldspar microphyric } \\
\text { basalt }\end{array}$ & None & None & $69.8-67.0$ & $30.0-32.3$ & $0.2-0.6$ & $64.2-56.2$ & $35.4-42.3$ & $0.4-1.5$ & $39.8-34$ & $45-48$ & $15.5-18$ & $\begin{array}{l}\text { Small plagioclase phenocrysts ( } 0.5 \\
\text { mm or less) set in groundmass of } \\
\text { felted plagioclase laths forming inter- } \\
\text { granular (fig. } 8 \text { ) and intersertal tex- } \\
\text { tures. Vesicles and calcite-filled } \\
\text { amygdules up to } 0.6 \mathrm{~mm}\end{array}$ \\
\hline $321-14-1,99-102$ & Aphyric basalt & & None & & & & $64.8-45.2$ & $34.7-54.1$ & $0.4-0.7$ & $37.6-32.5$ & $42.2-51.6$ & $20.2-15.8$ & $\begin{array}{l}\text { Texture idential to the groundmass } \\
\text { texture of Sample } 321-13-4,119-124 \\
\mathrm{~cm} \text { (fig. } 9 \text { ); Vesicles and amygdules } \\
\text { up to } 1.5 \mathrm{~mm} \text { across }\end{array}$ \\
\hline $321-14-2,127-130$ & $\begin{array}{l}\text { Fine-grained } \\
\text { feldspar phyric } \\
\text { basalt }\end{array}$ & $\begin{array}{l}\text { None } \\
\mathrm{N}\end{array}$ & None & 70.4 & 29.2 & 0.3 & $68.0-38.1$ & $31.6-60.8$ & $0.4-1.1$ & $35.4-30$ & $45.7-43.5$ & $18.8-26.5$ & $\begin{array}{l}\text { Texture similar to Sample 321-13-4, } \\
119-124 \mathrm{~cm} \text {; Vesicles and calcite- } \\
\text { filled vesicles } 1 \mathrm{~mm} \text { across }\end{array}$ \\
\hline 321-14-3, 93-96 & $\begin{array}{l}\text { Fine-grained } \\
\text { feldspar phyric } \\
\text { basalt }\end{array}$ & None & None & 68.7 & 31.0 & 0.3 & $67.9-46.1$ & $31.7-53.2$ & $0.4-0.7$ & $30-32$ & $50-47$ & $20-21$ & $\begin{array}{l}\text { Tex ture similar to Sample 321-13-4, } \\
119-124 \mathrm{~cm} \text {; Vesicles and amygdules } \\
1 \mathrm{~mm} \text { across }\end{array}$ \\
\hline $321-14-4,61-64$ & Aphyric basalt & & None & & & & $65.2-47.8$ & $34.3-51.1$ & $0.6-1.1$ & $37-28$ & $49.5-52$ & $13.5-20$ & $\begin{array}{l}\text { Texture similar to Sample } 321-14-1 \text {, } \\
99-102 \mathrm{~cm} \text {; Vesicles less than } 0.6 \mathrm{~mm} \\
\text { across }\end{array}$ \\
\hline
\end{tabular}

aIdentified by shipboard scientists. Numbering system that of Rhodes et al. (this volume).

$\mathbf{b}_{\text {Core and rim compositions. }}$

${ }^{c_{R} \text { Range of core compositions. }}$

dMost calcic core and most sodic rim compositions.

e Most magnesian and least magnesian augite. 
TABLE 2

Olivine Compositions in Selected Leg 34 Samples

\begin{tabular}{|c|c|c|c|c|c|c|c|c|c|c|c|c|c|c|}
\hline & \multirow{3}{*}{\multicolumn{2}{|c|}{$\begin{array}{c}\text { 319A-1-1, } \\
\text { 138-142 cm } \\
\text { Skeletal Grains }\end{array}$}} & \multicolumn{9}{|c|}{$319 \mathrm{~A}-3-4,18-21 \mathrm{~cm}$} & & & \\
\hline & & & \multicolumn{2}{|c|}{ Phenocryst } & \multicolumn{2}{|c|}{ Groundmass } & \multicolumn{3}{|c|}{ Phenocryst } & \multicolumn{2}{|c|}{ Groundmass } & & & \\
\hline & & & Core & $\operatorname{Rim}$ & Core & Rim & & & im & Core & $\operatorname{Rim}$ & & & \\
\hline $\mathrm{SiO}_{2}$ & 39.90 & 39.61 & 39.27 & 38.58 & 39.37 & 38.80 & 39 & & .26 & 38.88 & 38.06 & & & \\
\hline $\mathrm{FeO}$ & 13.93 & 13.93 & 16.40 & 21.28 & 18.44 & 18.36 & 13 & & 80 & 18.31 & 22.07 & & & \\
\hline $\mathrm{MnO}$ & 0.23 & 0.25 & 0.23 & 0.35 & 0.32 & 0.43 & & & .36 & 0.28 & 0.38 & & & \\
\hline $\mathrm{MgO}$ & 45.10 & 45.11 & 43.37 & 39.48 & 41.71 & 41.90 & & & .90 & 41.81 & 38.81 & & & \\
\hline $\mathrm{NiO}$ & 0.14 & 0.14 & 0.19 & 0.15 & 0.17 & 0.14 & & & .11 & 0.14 & 0.14 & & & \\
\hline $\mathrm{CaO}$ & 0.31 & 0.28 & 0.26 & 0.33 & 0.28 & 0.26 & & & .31 & 0.32 & 0.35 & & & \\
\hline Total & 99.61 & 99.32 & 99.72 & 100.17 & 100.29 & 99.89 & 99 & & .74 & 99.74 & 99.81 & & & \\
\hline \multirow[t]{4}{*}{ Fo mol \% } & 85 & 85 & 82 & 77 & 80 & 80 & 85 & 7 & & 80 & 76 & & & \\
\hline & \multicolumn{5}{|c|}{$319 \mathrm{~A}-3-5,37-41 \mathrm{~cm}$} & \multicolumn{9}{|c|}{$319 \mathrm{~A}-4-1,129-132 \mathrm{~cm}$} \\
\hline & \multirow{2}{*}{\multicolumn{3}{|c|}{$\begin{array}{l}\text { Grains in Troctolite } \\
\text { Fragment }\end{array}$}} & \multicolumn{2}{|c|}{ Phenocryst } & \multirow{2}{*}{\multicolumn{2}{|c|}{ Phenocryst }} & \multicolumn{2}{|c|}{ Phenocryst } & \multirow{2}{*}{\multicolumn{2}{|c|}{ Phenocryst }} & Phenocryst & \multirow[t]{2}{*}{ Groundmass } & \multirow[t]{2}{*}{ Groundmass } \\
\hline & & & & $\operatorname{Rim}$ & Core & & & Core & Rim & & & & & \\
\hline $\mathrm{SiO}_{2}$ & 39.21 & 39.34 & 39.93 & 40.74 & 40.49 & 39.8 & & 39.66 & 38.52 & & & 40.28 & 38.92 & 39.22 \\
\hline $\mathrm{FeO}$ & 15.76 & 16.05 & 13.63 & 13.36 & 10.57 & 12.9 & & 12.88 & 19.06 & & & 11.34 & 17.22 & 17.27 \\
\hline $\mathrm{MnO}$ & 0.22 & 0.20 & 0.21 & 0.30 & 0.21 & 0.1 & & 0.18 & 0.39 & & 20 & 0.18 & 0.26 & 0.27 \\
\hline $\mathrm{MgO}$ & 43.74 & 43.59 & 45.12 & 45.50 & 47.63 & 45.7 & & 45.76 & 40.46 & & & 46.66 & 42.30 & 42.23 \\
\hline $\mathrm{NiO}$ & 0.21 & 0.17 & 0.16 & 0.19 & 0.14 & 0.1 & & 0.19 & 0.14 & & 15 & 0.20 & 0.14 & 0.12 \\
\hline $\mathrm{CaO}$ & 0.31 & 0.29 & 0.30 & 0.33 & 0.24 & 0.2 & & 0.26 & 0.34 & & 28 & 0.24 & 0.29 & 0.36 \\
\hline Total & 99.45 & 99.64 & 99.35 & 100.42 & 99.28 & 99.1 & & 98.93 & 98.91 & & 59 & 98.90 & 99.13 & 99.47 \\
\hline \multirow[t]{4}{*}{ Fo mol \% } & 83 & 83 & 85.5 & 86 & 89 & 86 & & 86 & 79 & & & 88 & 81 & 81 \\
\hline & \multicolumn{5}{|c|}{$319 \mathrm{~A}-5-1,76-79 \mathrm{~cm}$} & \multicolumn{6}{|c|}{$319 \mathrm{~A}-7-1,119-122 \mathrm{~cm}$} & \multicolumn{3}{|c|}{$320 \mathrm{~B}-3-1,120-125 \mathrm{~cm}$} \\
\hline & \multirow{2}{*}{\multicolumn{2}{|c|}{ Phenocryst }} & \multirow{2}{*}{\multicolumn{2}{|c|}{ Groundmass }} & \multirow[t]{2}{*}{ Groundmass } & \multirow{2}{*}{\multicolumn{2}{|c|}{$\begin{array}{l}\text { Phenocryst } \\
\text { Core }\end{array}$}} & \multirow{2}{*}{\multicolumn{2}{|c|}{$\begin{array}{l}\text { Groundmass } \\
\text { Core }\end{array}$}} & Grou & Imass & Groundmass & Groundmass & s Groundmass \\
\hline & & & & & & & & & & Core & Rim & & & \\
\hline $\mathrm{SiO}_{2}$ & 39.23 & 39.14 & 39.1 & & 38.80 & 39.2 & & 39. & & 39.06 & 39.06 & 39.30 & 39.56 & 39.65 \\
\hline $\mathrm{FeO}$ & 15.35 & 16.84 & 17. & & 17.24 & 15. & & 14. & & 15.94 & 16.84 & 14.30 & 14.64 & 14.33 \\
\hline $\mathrm{MnO}$ & 0.26 & 0.30 & 0.2 & & 0.28 & 0.2 & & 0. & & 0.25 & 0.27 & 0.22 & 0.26 & 0.21 \\
\hline $\mathrm{MgO}$ & 44.22 & 42.78 & 41.5 & & 42.16 & 43. & & 44. & & 43.27 & 42.37 & 44.60 & 44.56 & 44.67 \\
\hline $\mathrm{NiO}$ & 0.14 & 0.15 & 0.1 & & 0.14 & 0. & & 0. & & 0.12 & 0.12 & 0.21 & 0.22 & 0.16 \\
\hline $\mathrm{CaO}$ & 0.26 & 0.30 & 0.2 & & 0.33 & 0. & & 0 . & & 0.32 & 0.37 & 0.35 & 0.27 & 0.30 \\
\hline Total & 99.46 & 99.51 & 99.5 & & 98.95 & 99. & & 99. & & 98.96 & 99.03 & 98.98 & 99.51 & 99.32 \\
\hline Fo mol \% & 83.5 & 82 & 81 & & 81 & 83. & & 84 & & 83 & 82 & 85 & 84 & 85 \\
\hline
\end{tabular}


TABLE 3

Plagioclase Compositions in Selected Leg 34 Samples

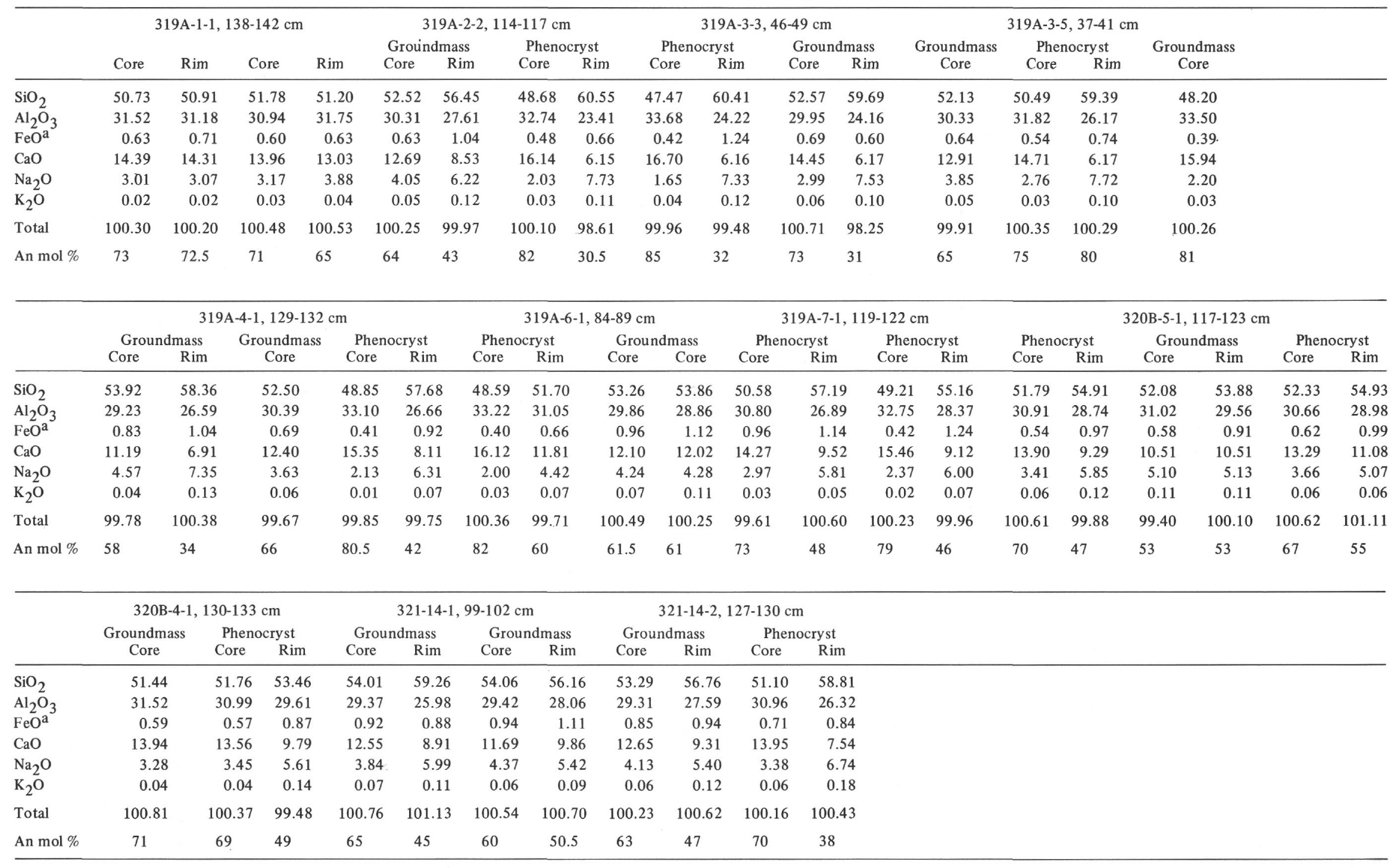

${ }^{\mathrm{a}} \mathrm{All}$ iron as $\mathrm{FeO}$. 
TABLE 4

Pyroxene Compositions in Selected Leg 34 Samples

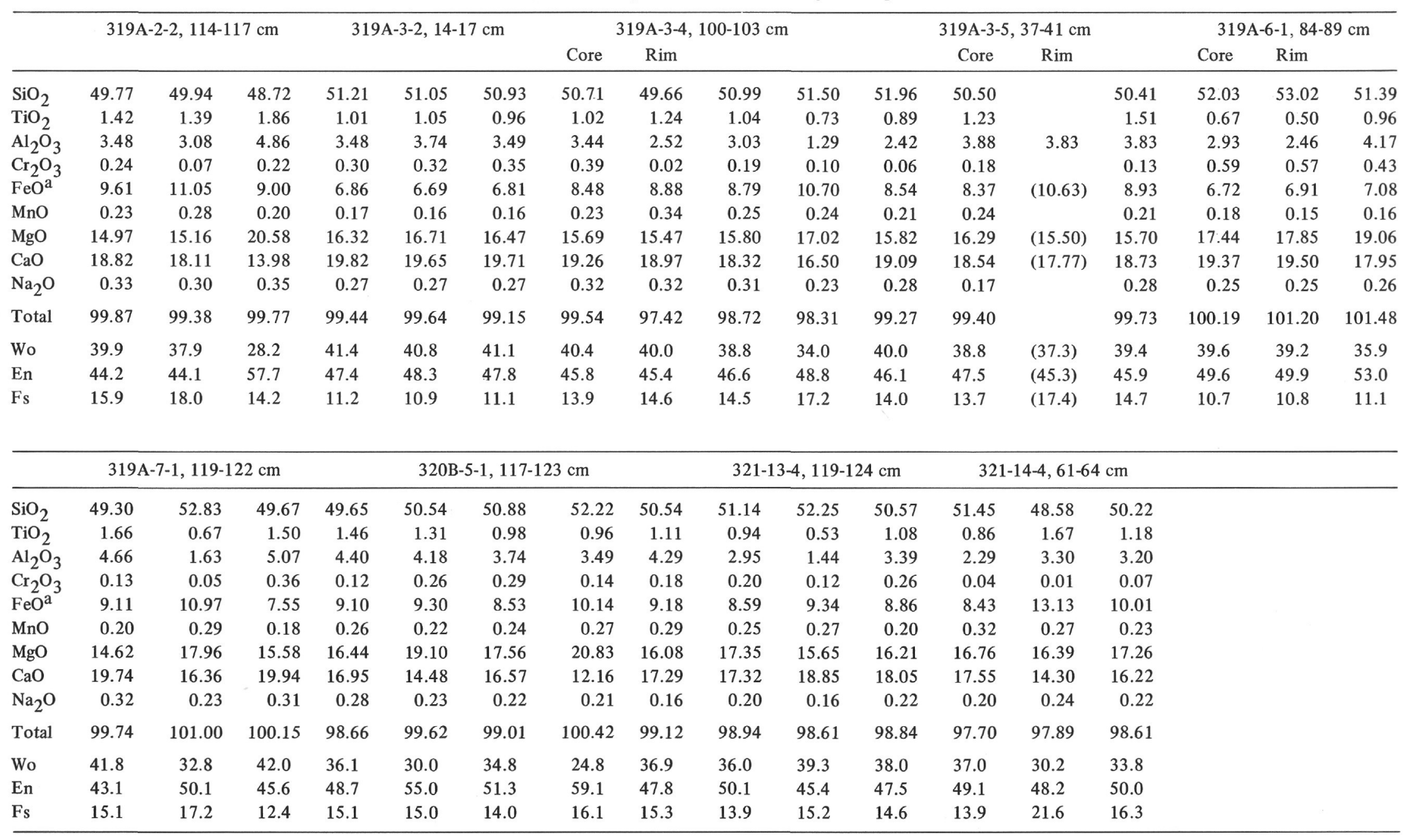

${ }^{\mathrm{a}} \mathrm{All}$ iron as $\mathrm{FeO}$. 
TABLE 5

Ilmenite and Titanomagnetite Compositions in Selected Leg 34 Samples

\begin{tabular}{|c|c|c|c|c|c|c|c|c|c|c|c|c|c|c|c|c|}
\hline \multicolumn{8}{|c|}{$319 \mathrm{~A}-3-2,127-130 \mathrm{~cm}$} & \multicolumn{4}{|c|}{$319 A-3-3,106-109 \mathrm{~cm}$} & & & & & \\
\hline $\mathrm{TiO}_{2}$ & 29.42 & 25.12 & 26.00 & 25.94 & 50.34 & 50.55 & 48.92 & 16.79 & 50.46 & 25.69 & 24.72 & & & & & \\
\hline $\mathrm{Al}_{2} \mathrm{O}_{3}$ & 1.89 & 2.00 & 1.55 & 1.41 & 0.11 & 0.00 & 0.06 & 1.69 & 0.10 & 2.08 & 2.03 & & & & & \\
\hline $\mathrm{Cr}_{2} \mathrm{O}_{3}$ & 0.04 & 0.04 & 0.02 & 0.03 & 0.02 & 0.00 & 0.02 & 0.04 & 0.00 & 0.03 & 0.03 & & & & & \\
\hline $\mathrm{FeO}^{\mathrm{a}^{\mathrm{J}}}$ & 65.36 & 67.95 & 69.29 & 67.29 & 49.37 & 47.24 & 49.31 & 74.35 & 49.28 & 66.70 & 67.89 & & & & & \\
\hline $\mathrm{MnO}$ & 0.60 & 0.62 & 0.78 & 0.80 & 0.98 & 2.84 & 0.74 & 0.58 & 0.61 & 0.65 & 0.76 & & & & & \\
\hline $\mathrm{MgO}$ & 0.36 & 0.65 & 0.30 & 0.27 & 0.62 & 0.26 & 0.53 & 0.24 & 0.84 & 1.01 & 0.48 & & & & & \\
\hline \multirow[t]{2}{*}{ Total } & 97.67 & 96.38 & 96.94 & 95.74 & 101.44 & 100.89 & 99.58 & 95.69 & 101.29 & 96.16 & 95.91 & & & & & \\
\hline & \multicolumn{4}{|c|}{$319 \mathrm{~A}-2-2,114-117 \mathrm{~cm}$} & \multicolumn{3}{|c|}{$319 \mathrm{~A}-3-2,14-17 \mathrm{~cm}$} & \multicolumn{2}{|c|}{$319 \mathrm{~A}-3-3,46-49 \mathrm{~cm}$} & \multicolumn{5}{|c|}{$319 \mathrm{~A}-3-4,18-21 \mathrm{~cm}$} & & \\
\hline $\mathrm{TiO}_{2}$ & 49.51 & 23.83 & 23.85 & 21.78 & 24.52 & 24.06 & 23.66 & 25.15 & 23.28 & 24.19 & 23.21 & 46.97 & 21.23 & 23.33 & & \\
\hline $\mathrm{FeO}^{\mathrm{a}}$ & 48.58 & 72.27 & 72.90 & 73.18 & 71.46 & 72.67 & 71.82 & 70.77 & 67.72 & 71.78 & 73.75 & 52.56 & 75.46 & 74.11 & & \\
\hline $\mathrm{MgO}$ & 0.05 & 0.51 & 0.33 & 0.22 & 0.49 & 0.44 & 0.52 & 0.83 & 0.86 & 0.49 & 0.33 & 0.33 & 0.17 & 0.29 & & \\
\hline \multirow[t]{2}{*}{ Total } & 98.94 & 96.61 & 97.08 & 95.18 & 96.47 & 97.17 & 96.00 & 96.75 & 91.86 & 96.46 & 97.29 & 99.86 & 96.86 & 97.73 & & \\
\hline & \multicolumn{4}{|c|}{$319 \mathrm{~A}-3-4,100-103 \mathrm{~cm}$} & \multicolumn{3}{|c|}{$319 A-3-5,37-41 \mathrm{~cm}$} & \multicolumn{4}{|c|}{$319 \mathrm{~A}-4-1,129-132 \mathrm{~cm}$} & \multicolumn{2}{|c|}{$\begin{array}{l}321-14-1, \\
99-102 \mathrm{~cm}\end{array}$} & \multicolumn{3}{|c|}{$321-14-2,127-130 \mathrm{~cm}$} \\
\hline $\mathrm{TiO}_{2}$ & 49.96 & 24.25 & 22.84 & 26.46 & 49.62 & 23.26 & 23.70 & 22.63 & 22.21 & 22.67 & 22.74 & 23.47 & 24.36 & 23.64 & 23.47 & 23.13 \\
\hline $\mathrm{FeO}^{\mathrm{a}}$ & 47.61 & 71.39 & 73.27 & 69.17 & 49.41 & 72.65 & 72.32 & 73.63 & 74.04 & 73.76 & 73.94 & 72.15 & 72.77 & 71.10 & 71.64 & 72.22 \\
\hline $\mathrm{MgO}$ & 1.55 & 0.95 & 0.69 & 0.96 & 0.44 & 0.64 & 0.38 & 0.67 & 0.50 & 0.47 & 0.31 & 0.45 & 0.55 & 0.46 & 0.62 & 0.34 \\
\hline Total & 99.13 & 96.59 & 96.80 & 96.59 & 99.47 & 96.55 & 96.40 & 96.93 & 96.75 & 96.90 & 96.99 & 96.07 & 97.68 & 95.20 & 95.73 & 95.69 \\
\hline
\end{tabular}

${ }^{\mathrm{a}} \mathrm{All}$ iron as $\mathrm{FeO}$. 
TABLE 6

Spinel Composition in Sample 320B-3-1, 120-125 cm

\begin{tabular}{lr}
\hline $\mathrm{TiO}_{2}$ & 1.08 \\
$\mathrm{Al}_{2} \mathrm{O}_{3}$ & 24.45 \\
$\mathrm{Cr}_{2} \mathrm{O}_{3}$ & 36.79 \\
$\mathrm{FeO}^{\mathrm{a}}$ & 23.73 \\
$\mathrm{MnO}$ & 0.22 \\
$\mathrm{MgO}$ & 13.32 \\
Total & 99.59 \\
\hline
\end{tabular}

${ }^{\text {a All iron as FeO. }}$

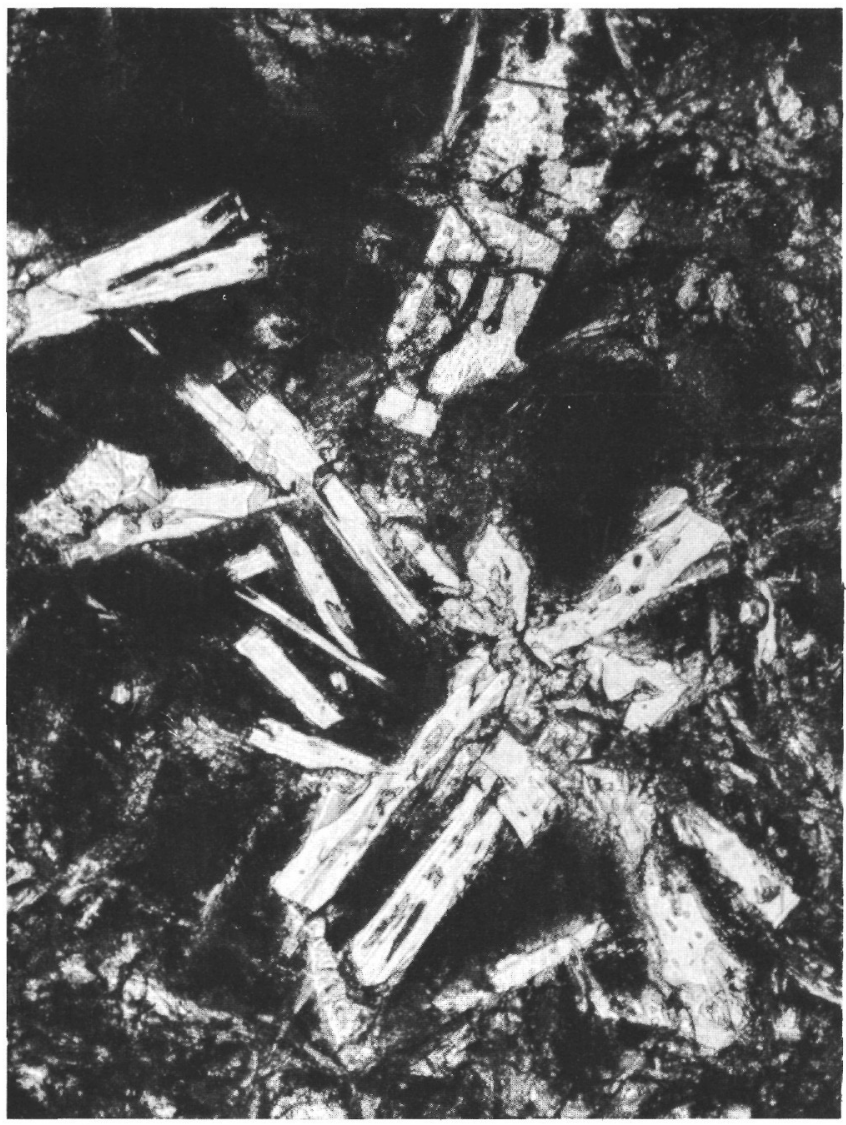

Figure 1. Skeletal olivine (top of photograph) and glomerocrysts of plagioclase laths and anhedral augite set in cryptocrystalline groundmass. $2 \times 2.3 \mathrm{~mm}$. Sample $319 A-1-1$, $138-142 \mathrm{~cm}$.

than those in basalts at Hole 319A. Core compositions of groundmass plagioclases in Site 321 basalts are more sodic than those in samples from Hole $319 \mathrm{~A}$, and they have larger orthoclase contents (Table 1). These petrographic differences are not attributable to differences in cooling history between the basalts at the two sites. Rather, site 321 basalts are inferred to be more alkali rich than those in Hole 319A and to have had different crystallization histories. Although the core compositions of plagioclase phenocrysts in samples from Hole 320B are not as calcic as those of the most anorthite-rich plagioclases at Hole 319A, the rocks from both holes are similar in phase compositions (Table 1). The generally smaller range of phase zoning in rocks from Hole $320 \mathrm{~B}$ reflects faster cooling.

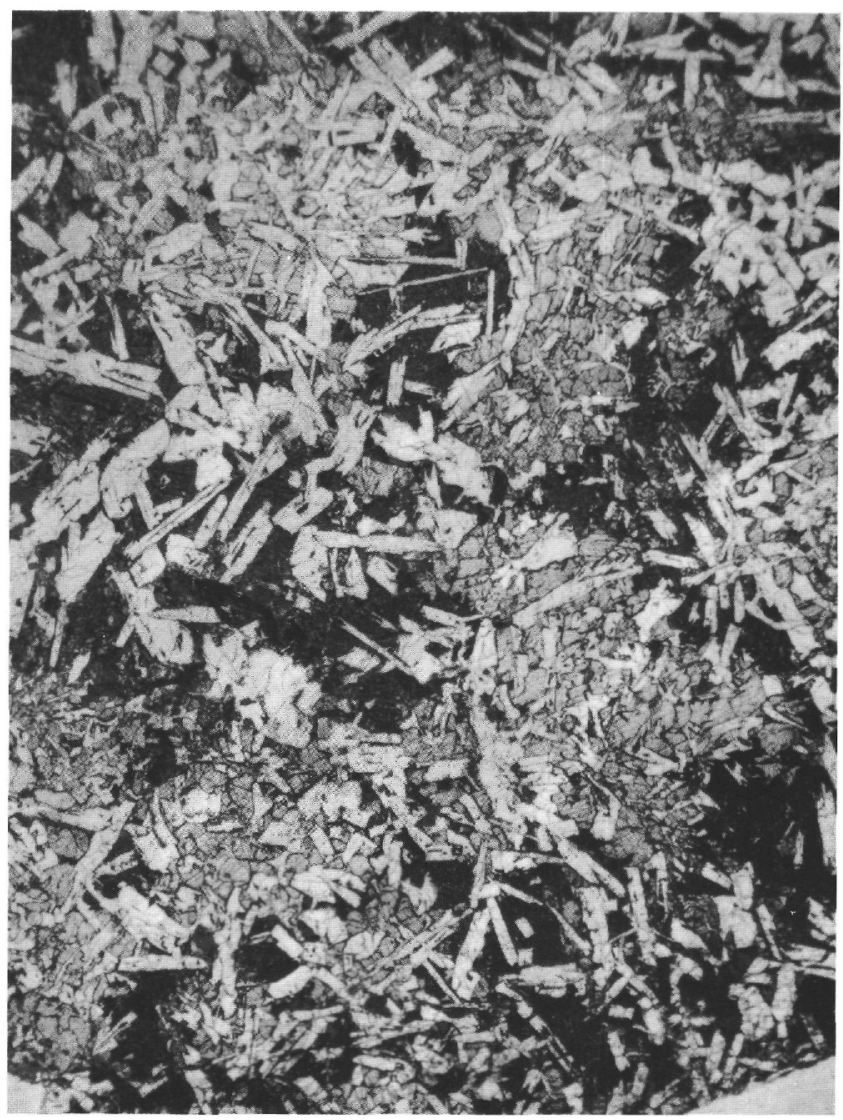

Figure 2. Poikilophitic and intersertal texture typical of those in medium-grained basalts at Hole 319A. $1 \times 1.3$ cm. Sample 319A-3-3, 46-49 cm.

At each site, basalts from different cooling units and within individual cooling units have similar parageneses, except for crystallization of olivine. The absence of olivine phenocrysts from some of our samples from the thick cooling unit 3 (Table 1) may be due to crystal settling. Our companion paper (Rhodes et al., this volume) indicates that basalts at Site 319 are tholeiitic in composition rather than transitional to alkaline in affinity, as inferred in the preliminary report by the shipboard scientists from the occurrence of groundmass olivine. In the thick cooling unit (3, Table 1) groundmass olivine is absent from rocks at the center of the unit. As noted by Evans and Moore (1968), persistence of groundmass olivine in a tholeiite is favored by rapid cooling and hence is an unsatisfactory index of magma type.

Our petrographic observations suggest that basalts at Sites 319 and 320 are plagioclase-(olivine) tholeiites, while those at Site 321 are more fractionated tholeiites. We conclude that basalts from all three sites are oceanic tholeiites and products of ocean ridge volcanism.

\section{ACKNOWLEDGMENTS}

C.H. Donaldson thanks the Natural Environment Research Council of the United Kingdom for financial support and the Lunar Science Institute for a Visiting Graduate Fellowship. Mike Rhodes kindly reviewed the manuscript. The Lunar Science Institute is operated by the Universities Space Research 


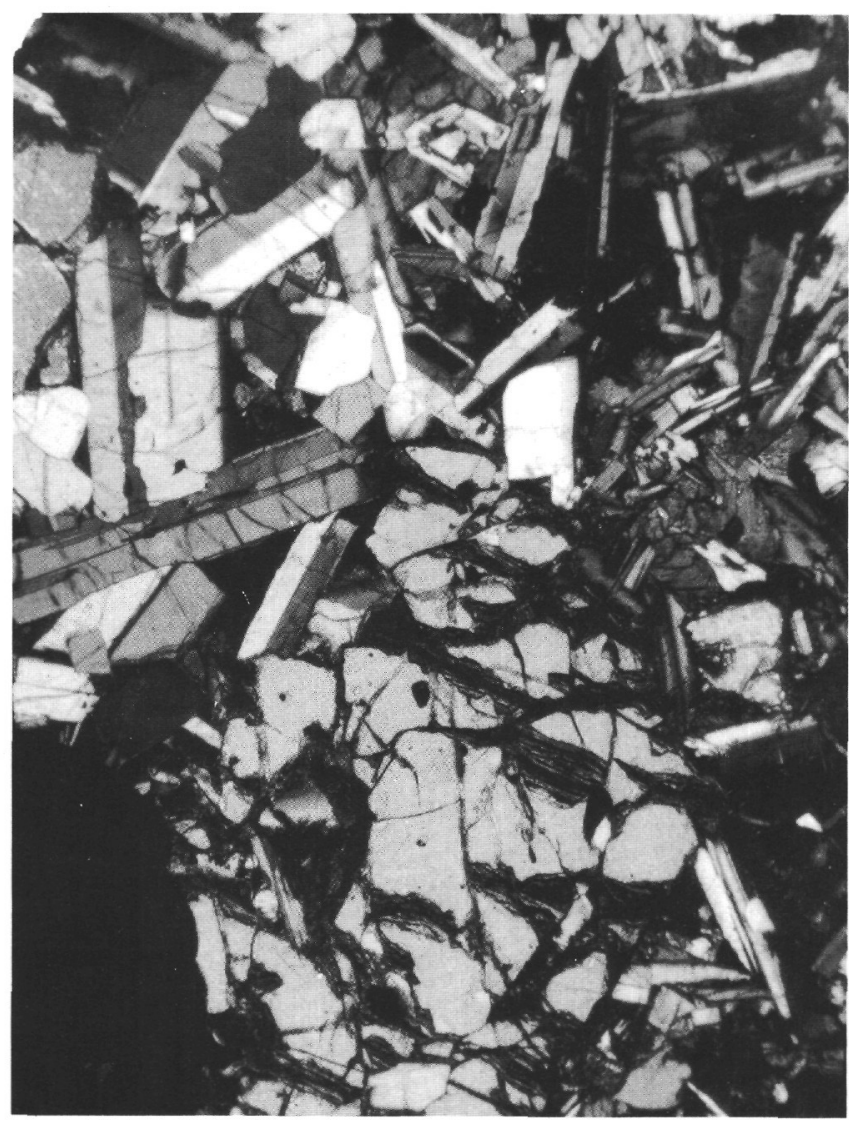

Figure 3. Troctolite xenolith in olivine-feldspar phyric basalt. Large olivine crystal poikilitically encloses plagioclase crystals (bottom of photograph). Groundmass (at right of photograph) consists of plagioclase and augite. $5.2 \times 5.5 \mathrm{~mm}$. Polarized light. Sample 319A-3-5, 37-41 $\mathrm{cm}$.

Association under Contract No. NSR-09-051-001 with NASA. This paper is Lunar Science Institute Contribution No. 198.

\section{REFERENCES}

Evans, B.W. and Moore, J.G., 1968. Mineralogy as a function of depth in the Prehistoric Makaopuhi tholeiitic lava lake, Hawaii: Contrib. Mineral. Petrol., v. 17, p. 97.

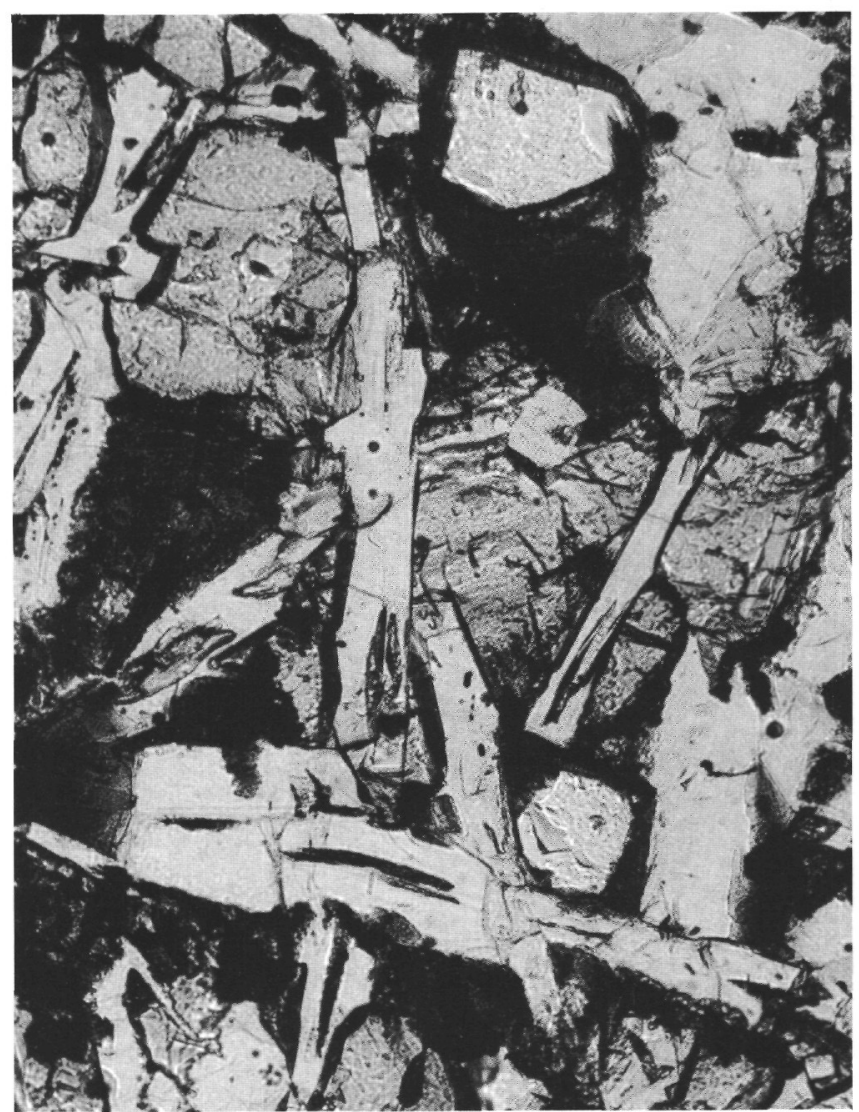

Figure 4. Groundmass olivine crystals (top center and lower center) trapped between plagioclase laths (white) which are subophitically enclosed by augite (gray). Note the dark rim of smectite around the olivine. $2.1 \times 2.3$ mm. Sample 319A-4-1, 129-132 cm.

Hart, S.R., et al., 1974. Oceanic basalts and the Nazca plate: Geotimes, v. 19, p. 20-24.

Muir, I.D., 1951. The clinopyroxenes of the Skaergaard intrusion, eastern Greenland: Min. Mag., v. 29, p. 690. 


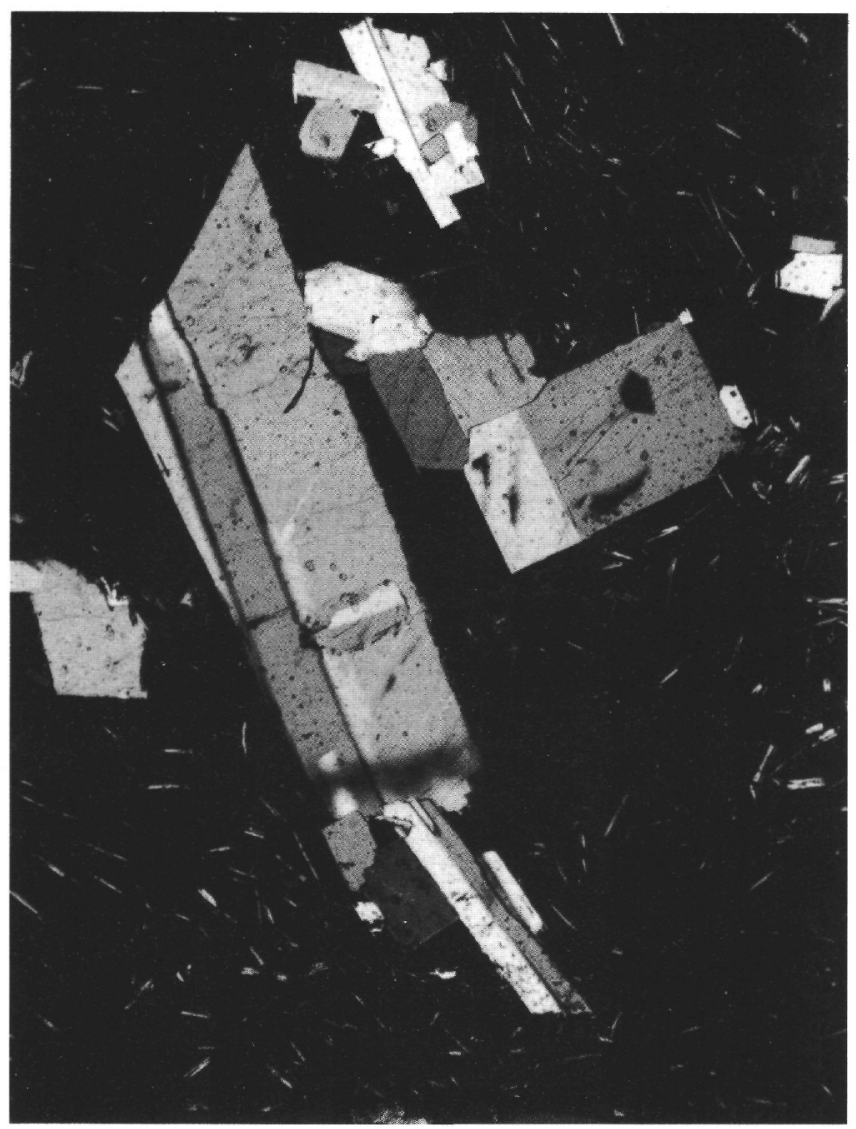

Figure 5. Glomerocrysts of plagioclase set in cryptocrystalline groundmass. $4 \times 4.3 \mathrm{~mm}$. Polarized light. Sample $319 A-6-1,84-89 \mathrm{~cm}$.

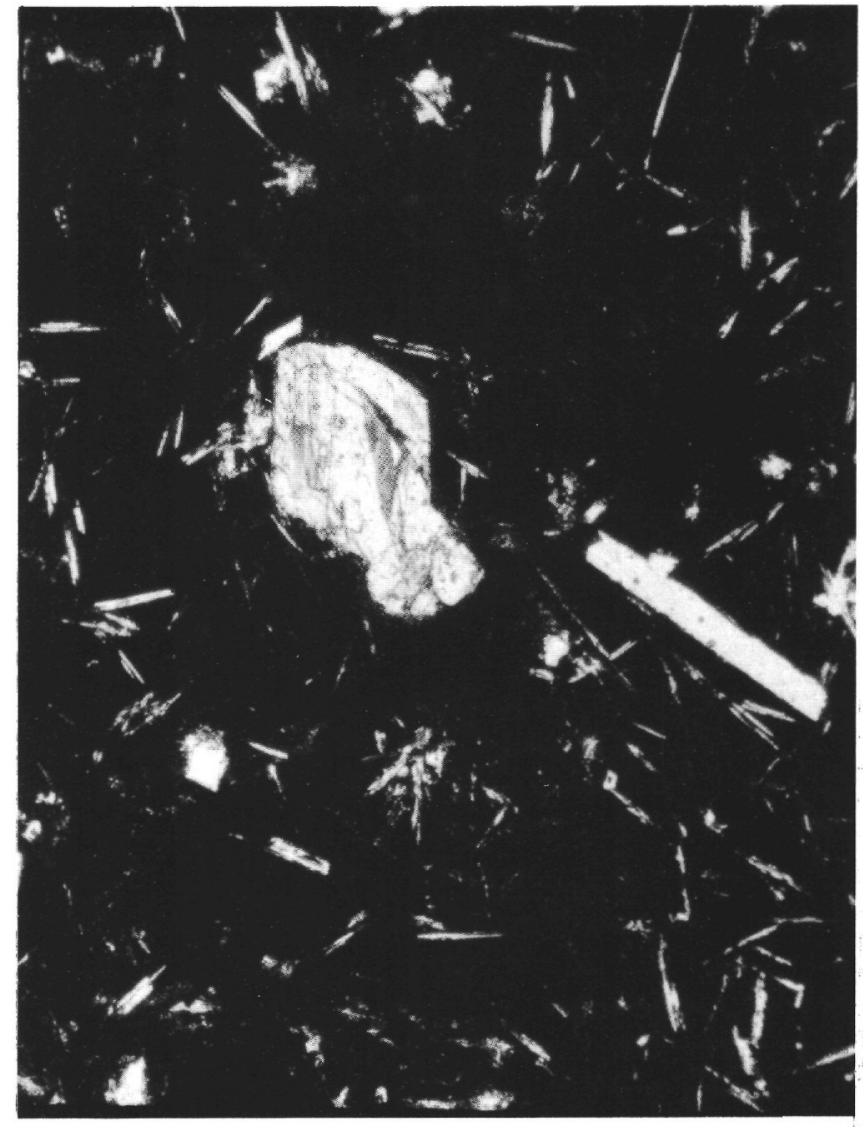

Figure 6. Pyroxene microphenocryst and plagioclase lath: set in a matrix of skeletal plagioclase laths and cryptocrystalline material. $1.5 \times 1.7 \mathrm{~mm}$. Sample 319A-6-1 $84-89 \mathrm{~cm}$. 


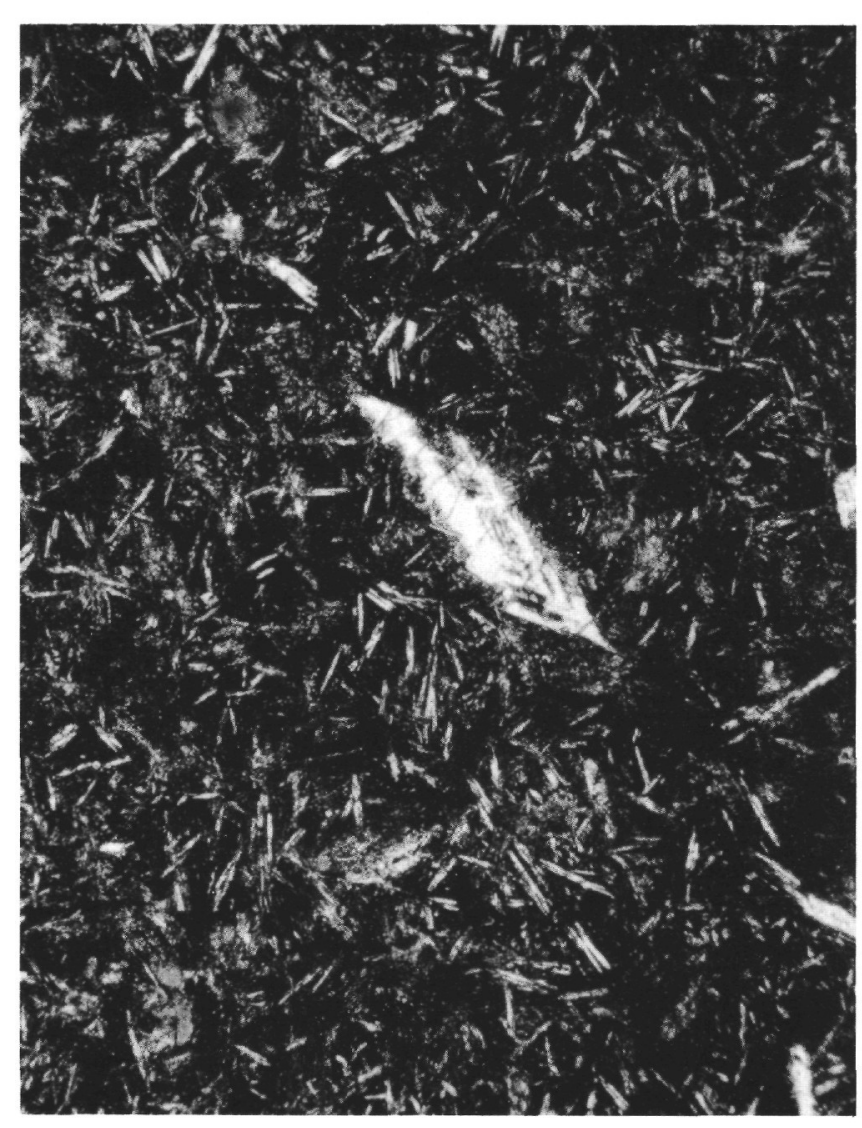

Figure 7. Skeletal plagioclase microphenocryst set in groundmass of felted plagioclase and cryptocrystalline material. $2 \times 2.2 \mathrm{~mm}$ across. Sample 320B-4-1, 130-133 cm.

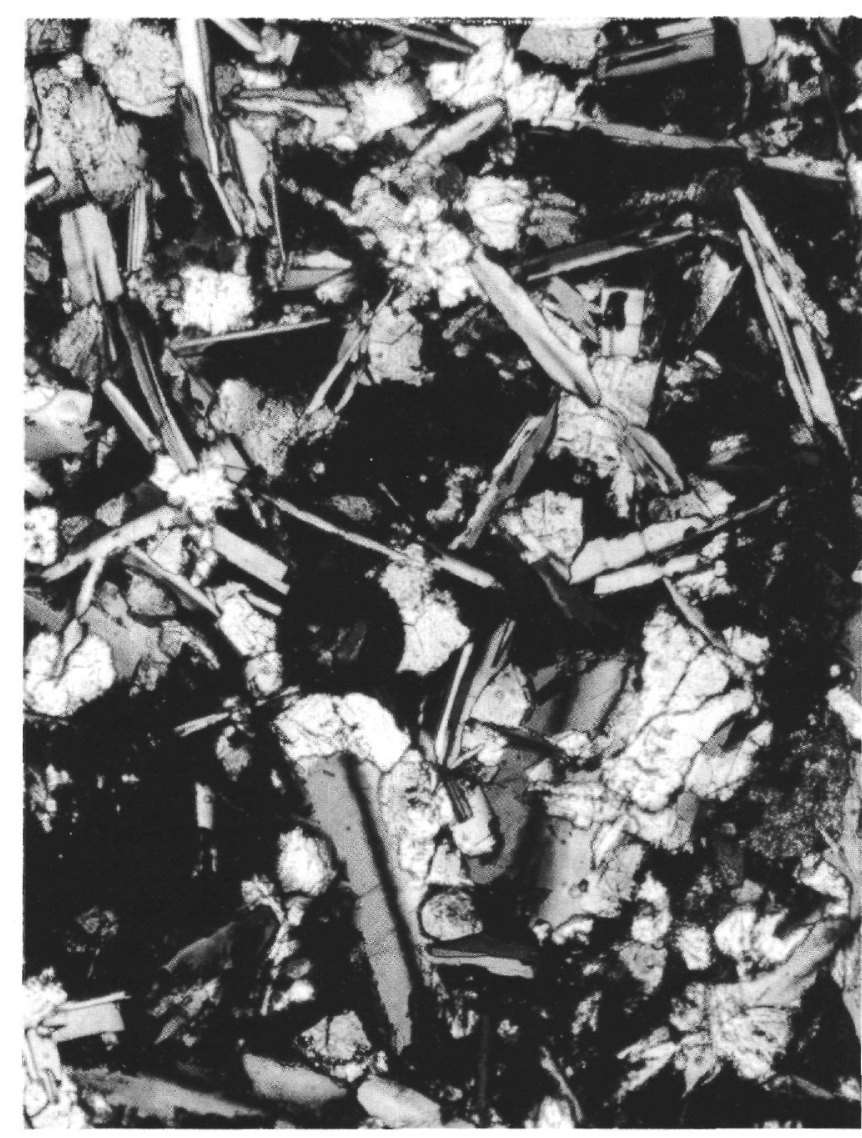

Figure 8. Intergranular texture of pyroxene and plagioclase in aphyric basalt. $1 \times 1.2 \mathrm{~mm}$. Polarized light. Sample 321-14-4, 61-64 cm. 


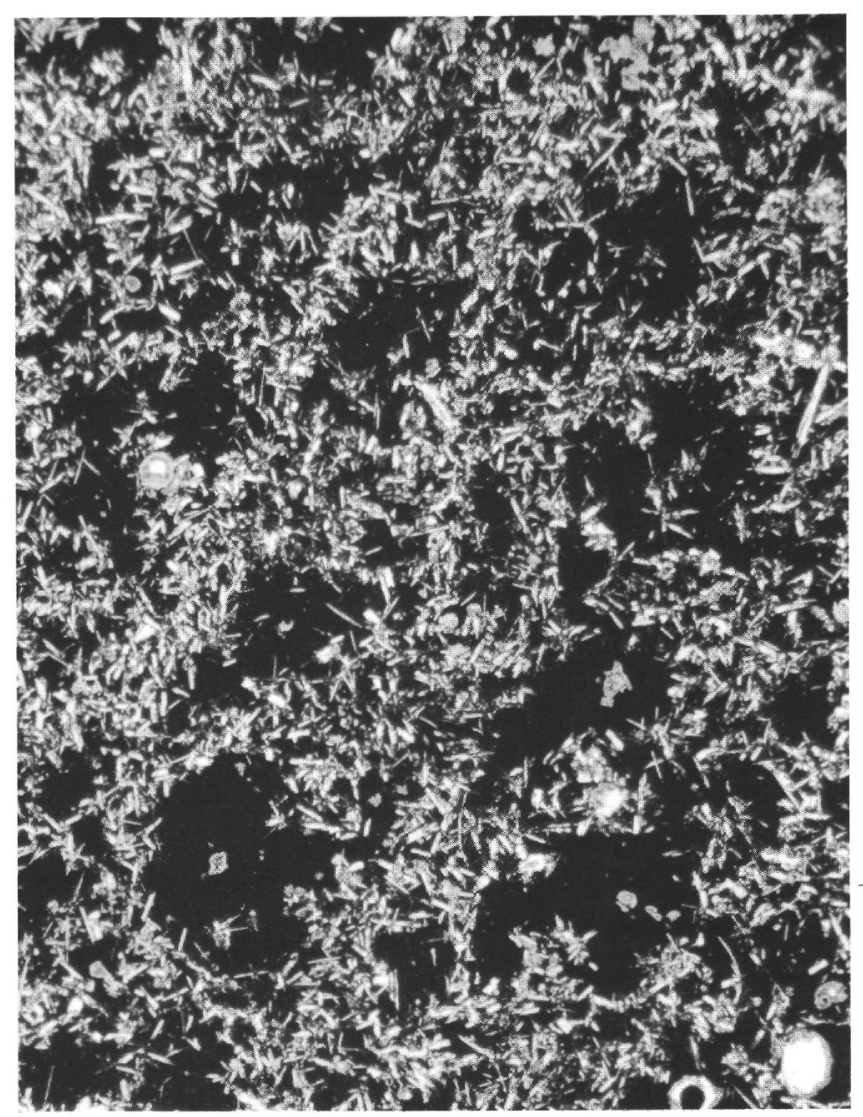

Figure 9. Intergranular and intersertal textures in groundmass of fine-grained feldspar phyric basalt. $5.2 \times 5.5$ mm. Sample 321-13-4, 119-124 cm.

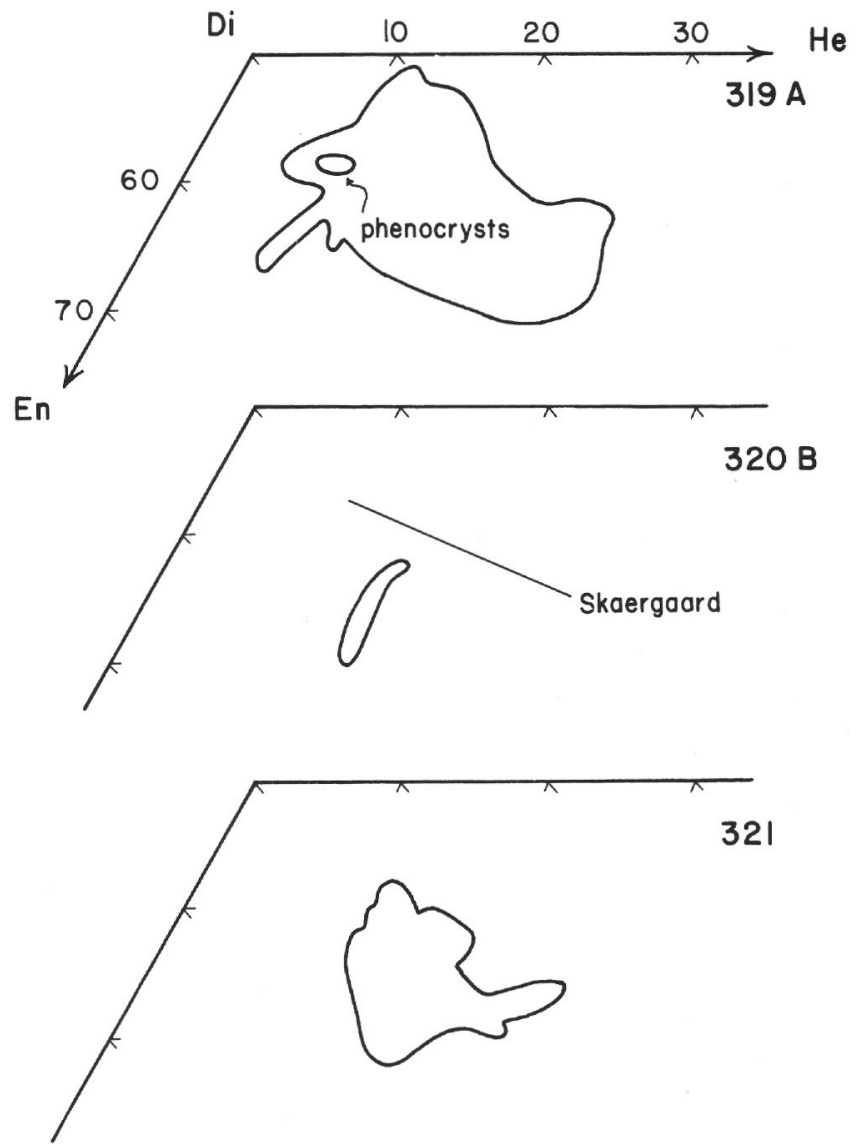

Figure 10. Summary of pyroxene compositions in samples from Holes $319 \mathrm{~A}, 320 \mathrm{~B}$, and 321 plotted in the $\mathrm{Di}-\mathrm{He}$ En-Fs quadrilateral. Line on diagram for Hole $320 B$ shows the trend of early crystallizing augite in the Skaergaard intrusion (Muir, 1941). 